\title{
Teeth Classification Based on Haar Wavelet Transform and Support Vector Machine
}

\author{
Fangyuan $\mathrm{Liu}^{1, *}$, Zhi $\mathrm{Li}^{2}$ and Wagner Quinn ${ }^{3}$ \\ ${ }^{1}$ Jiangsu Key Laboratory of 3D Printing Equipment and Manufacturing, Nanjing, Jiangsu 210042, China \\ ${ }^{2}$ Department of Stomatology, Jinling Hospital, School of Medicine, Nanjing University, 210002, Nanjing, Jiangsu, China \\ ${ }^{3}$ Department of Computer Science, University College Cork, Ireland \\ ${ }^{*}$ Corresponding author
}

\begin{abstract}
To improve the efficiency of stomatology practitioners, this paper proposed a novel teeth type classification method. Our method was based on three successful components: Haar wavelet transform, principal component analysis, and support vector machine. We create a 120 -image dataset, with 30 images for incisor, canine, premolar, and molar. The results showed our method achieved an overall classification accuracy of $81.83 \pm 1.79 \%$, better than decision tree and multilayer perceptron methods.
\end{abstract}

Keyword-Haar wavelet transform; support vector machine; principal component analysis

\section{INTRODUCTION}

It is well known that teeth are one of the most important organs in the human body, which help chew food, control pronunciation and keep healthy. Learning and acquiring dental information is closely watched by researchers from the medical and academic fields. At present, dental information is collected and recorded by the staff through the oral head imaging equipment. In practical application, CT technique is most beneficial to identify, diagnose, and treat teeth. CT has the advantages of low ray volume, short scan time, high image clarity and simple operation, so as to provide accurate data recording and reduce the experimental cost.

In recent years, researchers have proposed various methods for tooth recognition and classification. Yoke-San, Xin [1] introduced a classification system based on the iterative closest point algorithm(ICP) to handle four types of teeth. The system used PCA extracted eigenteeth feature, projected datasets into the tooth geometric space and then classify tooth type by KNN. Ghodsi and Faez [2] created an automated dental identification system (AIDS) which applied Zernike moment(ZM) to extract feature and calculate Euclidian distance in the match phase. The method reduced search space, increased matching certainty by voting mechanism and ZM ensured the stability of experimental results. Al-sherif, Guo [3] established a method contains two stages for identifying bitewing dental images: (i) Orthogonal Locality Preserving Projection(OLPP) projected Laplacianteeth spaces to complete the initial classification; (ii) validate class and assign number based on teeth neighborhood rules, the classification accuracy reached 89\%. Hosntalab, Zoroofi [4] combined the traditional segmentation techniques with wavelet-Fourier descriptors(WFDs) to select the most effective eigenvector, and then used feed-forward neural network to classify tooth images. Pushparaj, Gurunathan [5] utilized support vector machine and universal numbering system to recognize teeth and number of teeth respectively. The key part of this approach is feature extraction which is aimed at individual teeth identification. PPED vectors, geometric features and regional descriptors are used in the process of feature extraction. The results show that the method is feasible and effective, and the average accuracy rate is $92.65 \%$. Yeesarapat, Auephanwiriyakul [6] adapted fuzzy C-means clustering algorithm(FCM) based on multiple features(the pixel values of RGB and HIS) to classify dental fluorosis. FCM determines tooth status by cyclic updating membership values and prototypes until stable. Tangel, Fatichah [7] presented a fuzzy inference system to achieve four categories of dental images. This system used Mamdani type FIS with centroid defuzzification method which employed multiple fuzzy attributes and the selected threshold is 0.15 . This approach can deal with blurred tooth images and guaranteed the robustness of the classification, the overall classification accuracy reaches 82.51\%. Karthick and Harikumar [8] analyzed the performance of Naïve Bayes and SVM in the classification of five dental diseases. Before classifying the tooth images, they preprocessed through Median filter, segmented and extracted GLCM features by FCM algorithm. Veeraprasit and Phimoltares [9] described a model to recognize the tooth images by using the mixed feature. The researchers fused global features (singular values and color histogram) and local features (teeth width), and then applied MLP with Levenberg-Marquardt algorithm to determine the type of tooth effectively. Finally, the accuracy rate of the experiment is about $93.6 \%$, which is superior to Naïve Bayes and k-Nearest Neighbor methods.

Based on the above studies on classification methods, the potential for various learning is getting better in various fields at the same time. In this paper, support vector machine is proposed to classify four kinds of dental images: central incisor, lateral incisor, canine and premolar. The methodology adopted in this study is described in Section 2. The material used in the experiment is shown in Section 3. In Section 4, we analyzed the experiments and results in detail, which is compared with the current methods. The last part is Section 5 which includes conclusion and the prospects we expect.

\section{METHOD}

Firstly, we utilize histogram equalization technique [10] to enhance the data of dental images. Histogram equalization method has the following advantages: (i) increased the contrast; (ii) improved the changes of gray tone; (iii) enhanced the 
sharpness of the image, so that we can obtain high-quality sample data.

Next, Haar wavelet transform is one of the most popular wavelet transform in the field of wavelet analysis [11-14]. It can guarantee the integrity of image information because it gets the global information while recording the local information. The basic Haar wavelet function [15] is defined as Formula (1) and the scaling function is defined as Formula (2). In Formula (2), $j$ is a scaling factor and $i$ is a translation parameter. In the future, we shall test the performance of biorthogonal wavelet [16, 17].

After extracting the four-level wavelet features, we used PCA to decrease the feature dimension. This way can reduce the number of features, noise and redundancy, and the likelihood of excessive fitting.

$$
\begin{gathered}
x(a)=\left\{\begin{array}{cc}
1, & 0 \leq a \leq 1 / 2 \\
-1, & 1 / 2 \leq a \leq 1 \\
0, & \text { otherwise }
\end{array}\right. \\
x^{j}(a)=x\left(2^{j} a-i\right), i=0, \cdots,\left(2^{j}-1\right)
\end{gathered}
$$

Finally, we employ an effective classification learning model, support vector machine (SVM), to classify four types of dental images.

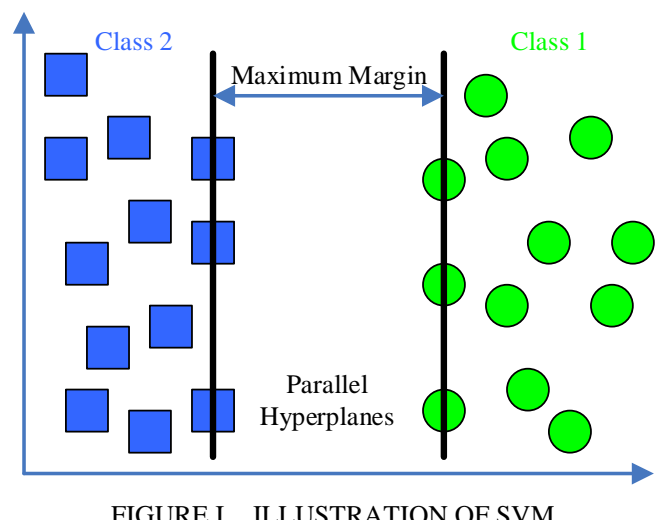

As far, SVM has been widely used in medical detection, text processing [18], speech recognition [19], and so on. SVM has been reported to be superior to the traditional classification method, such as BPNN [20], RBF network [21], k-Nearest Neighbors algorithm. Figure 1 gives an illustration of SVM, which finds several samples called "support vectors" [22-24] and created hyper-planes based on them [25-30].

\section{DATASET}

We used the ISO standard dental notation. Take upper right as an example, 11 and 12 are incisors; 13 is canine; 14 and 15 are premolars; 16, 17, and 18 are molars. Some samples of the dataset are listed in Figure 1. The images were obtained by participating hospitals. In total, we have a 120-image dataset, which contains 30 incisors, 30 canines, 30 premolars, and 30 molars. Figure 2 gives an example of each teeth type.

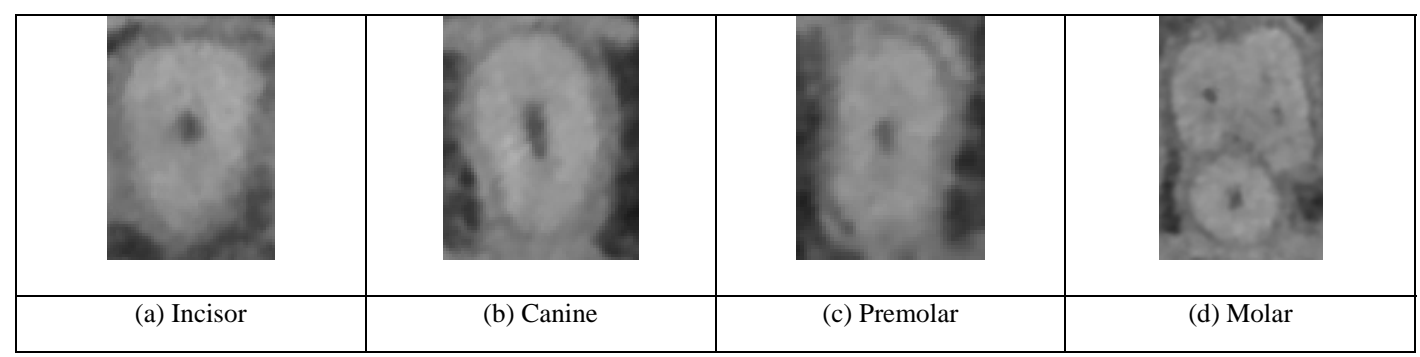

FIGURE II. SAMPLES OF DATASET

\section{EXPERIMENTS AND RESULTS}

This proposed "HWT_PCA_SVM" method was implemented 10 times. Within each time, a 5-fold cross validation was used. That means there are 6 image for each tooth type within each fold. The classification results in terms of sensitivity were given in Tables 1 . The whole accuracy of all four classes were given in Table 2.
TABLE I. SENSITIVITY OF EACH CLASS

\begin{tabular}{|c|c|c|c|c|}
\hline & Incisor & Canine & Premolar & Molar \\
\hline R1 & 76.67 & 86.67 & 73.33 & 90.00 \\
\hline R2 & 86.67 & 86.67 & 76.67 & 86.67 \\
\hline R3 & 83.33 & 76.67 & 90.00 & 86.67 \\
\hline R4 & 76.67 & 76.67 & 86.67 & 83.33 \\
\hline R5 & 83.33 & 83.33 & 80.00 & 83.33 \\
\hline R6 & 86.67 & 80.00 & 83.33 & 76.67 \\
\hline R7 & 80.00 & 80.00 & 86.67 & 86.67 \\
\hline R8 & 86.67 & 83.33 & 83.33 & 70.00 \\
\hline R9 & 80.00 & 80.00 & 80.00 & 83.33 \\
\hline R10 & 86.67 & 76.67 & 73.33 & 76.67 \\
\hline Average & $82.67 \pm 4.10$ & $81.00 \pm 3.87$ & $81.33 \pm 5.71$ & $82.33 \pm 6.10$ \\
\hline
\end{tabular}


TABLE II. ACCURACY OF ALL FOUR CLASSES

\begin{tabular}{|c|c|c|c|c|c|c|}
\hline & F1 & F2 & F3 & F4 & F5 & Total \\
\hline R1 & 70.83 & 87.50 & 91.67 & 83.33 & 75.00 & 81.67 \\
\hline R2 & 87.50 & 83.33 & 87.50 & 83.33 & 79.17 & 84.17 \\
\hline R3 & 79.17 & 87.50 & 79.17 & 91.67 & 83.33 & 84.17 \\
\hline R4 & 75.00 & 66.67 & 91.67 & 75.00 & 95.83 & 80.83 \\
\hline R5 & 75.00 & 83.33 & 83.33 & 87.50 & 83.33 & 82.50 \\
\hline R6 & 79.17 & 79.17 & 83.33 & 79.17 & 87.50 & 81.67 \\
\hline R7 & 79.17 & 87.50 & 95.83 & 62.50 & 91.67 & 83.33 \\
\hline R8 & 75.00 & 66.67 & 95.83 & 75.00 & 91.67 & 80.83 \\
\hline R9 & 79.17 & 75.00 & 87.50 & 83.33 & 79.17 & 80.83 \\
\hline R10 & 70.83 & 70.83 & 91.67 & 83.33 & 75.00 & 78.33 \\
\hline Average & & & & & & $81.83 \pm 1.79$ \\
\hline
\end{tabular}

We observe that the sensitivities of incisor, canine, premolar and molar are $82.67 \pm 4.10 \%, 81.00 \pm 3.87 \%, 81.33 \pm 5.71 \%$, and $82.33 \pm 6.10 \%$, respectively. The whole accuracy was $81.83 \pm$ $1.79 \%$. This demonstrates the effectiveness of HWT, PCA, and SVM. Except HWT, there are many other excellent feature descriptors, such as fractional Fourier transform [31], etc.

In addition, we compared our method with traditional decision tree (DT) [32], and multilayer perceptron (MLP) [33]. All the methods were implemented by 10 runs, and the results are shown in Table 3.

\section{TABLE III. ALGORITHM COMPARISON}

\begin{tabular}{|c|c|}
\hline Method & Accuracy \\
\hline DT [32] & $73.83 \pm 2.05$ \\
\hline MLP [33] & $76.25 \pm 3.54$ \\
\hline SVM (Proposed) & $81.83 \pm 1.79$ \\
\hline
\end{tabular}

The DT [32] obtained an overall accuracy of $73.83 \pm 2.05 \%$. MLP [33] obtained an overall accuracy of $76.25 \pm 3.54 \%$. The details of two basis algorithms were show in Table 4. Finally, the proposed SVM method could yield the greatest accuracy of $81.83 \pm 1.79 \%$.

TABLE IV. ACCURACY OF DT [32] AND MLP [33]

\begin{tabular}{|c|c|c|c|c|c|c|}
\hline DT [32] & F1 & F2 & F3 & F4 & F5 & Total \\
\hline R1 & 75.00 & 75.00 & 75.00 & 62.50 & 83.33 & 74.17 \\
\hline R2 & 66.67 & 70.83 & 70.83 & 83.33 & 66.67 & 71.67 \\
\hline R3 & 79.17 & 79.17 & 62.50 & 79.17 & 79.17 & 75.83 \\
\hline R4 & 83.33 & 70.83 & 70.83 & 83.33 & 70.83 & 75.83 \\
\hline R5 & 75.00 & 62.50 & 66.67 & 66.67 & 79.17 & 70.00 \\
\hline R6 & 66.67 & 70.83 & 70.83 & 79.17 & 79.17 & 73.33 \\
\hline R7 & 83.33 & 62.50 & 79.17 & 79.17 & 75.00 & 75.83 \\
\hline R8 & 58.33 & 91.67 & 75.00 & 87.50 & 66.67 & 75.83 \\
\hline R9 & 75.00 & 75.00 & 62.50 & 75.00 & 79.17 & 73.33 \\
\hline R10 & 75.00 & 66.67 & 79.17 & 83.33 & 58.33 & 72.50 \\
\hline Average & & & & & & $73.83 \pm 2.05$ \\
\hline MLP [33] & F1 & F2 & F3 & F4 & F5 & Total \\
\hline R1 & 91.67 & 95.83 & 75.00 & 70.83 & 62.50 & 79.17 \\
\hline R2 & 62.50 & 75.00 & 91.67 & 75.00 & 83.33 & 77.50 \\
\hline R3 & 70.83 & 79.17 & 87.50 & 79.17 & 75.00 & 78.33 \\
\hline R4 & 79.17 & 83.33 & 95.83 & 83.33 & 66.67 & 81.67 \\
\hline R5 & 70.83 & 75.00 & 75.00 & 62.50 & 62.50 & 69.17 \\
\hline R6 & 75.00 & 62.50 & 75.00 & 87.50 & 83.33 & 76.67 \\
\hline R7 & 87.50 & 62.50 & 83.33 & 62.50 & 66.67 & 72.50 \\
\hline R8 & 83.33 & 58.33 & 83.33 & 70.83 & 79.17 & 75.00 \\
\hline R9 & 79.17 & 79.17 & 75.00 & 79.17 & 75.00 & 77.50 \\
\hline R10 & 83.33 & 66.67 & 70.83 & 79.17 & 75.00 & 75.00 \\
\hline Average & & & & & & $76.25 \pm 3.54$ \\
\hline Alth0ugh
\end{tabular}

Although SVM obtained good results, we shall try to collect more data than used in this study, and tested the effect of deep learning, such as convolutional neural network [34-36], deep belief network [37], and autoencoder [38, 39].

\section{V.CONCLUSIONS}

In this study, we proposed a novel method based on Haar wavelet transform, principal component analysis, and support vector machine. The simulation experiments validated the effectiveness of our method.

\section{REFERENCES}

[1] Yoke-San, W., et al. An approach for single tooth classification and identification. in 2015 IEEE 10th Conference on Industrial Electronics and Applications (ICIEA). 2015. p. 1698-1702

[2] Ghodsi, S.B. and K. Faez. A novel approach for matching of dental radiograph image using Zernike moment. in 2012 IEEE International Conference on Computer Science and Automation Engineering (CSAE). 2012. p. 303-306

[3] Al-sherif, N., G. Guo, and H.H. Ammar. Automatic Classification of Teeth in Bitewing Dental Images Using OLPP. in 2012 IEEE International Symposium on Multimedia. 2012. p. 92-95

[4] Hosntalab, M., et al. Automated Dental Recognition in MSCT Images for Human Identification. in 2009 Fifth International Conference on Intelligent Information Hiding and Multimedia Signal Processing. 2009. p. 1318-1321

[5] Pushparaj, V., et al. An effective numbering and classification system for dental panoramic radiographs. in 2013 Fourth International Conference on Computing, Communications and Networking Technologies (ICCCNT). 2013. p. 1-8

[6] Yeesarapat, U., et al. Dental fluorosis classification using multiprototypes from fuzzy C-means clustering. in 2014 IEEE Conference on Computational Intelligence in Bioinformatics and Computational Biology. 2014. p. $1-5$

[7] Tangel, M.L., et al. Dental classification for periapical radiograph based on multiple fuzzy attribute. in 2013 Joint IFSA World Congress and NAFIPS Annual Meeting (IFSA/NAFIPS). 2013. p. 304-309

[8] Karthick, G. and R. Harikumar. Comparative performance analysis of Naive Bayes and SVM classifier for oral X-ray images. in 2017 4th International Conference on Electronics and Communication Systems (ICECS). 2017. p. 88-92

[9] Veeraprasit, S. and S. Phimoltares. Hybrid feature-based teeth recognition system. in 2011 IEEE International Conference on Imaging Systems and Techniques. 2011. p. 302-305

[10] Wu, X., Smart detection on abnormal breasts in digital mammography based on contrast-limited adaptive histogram equalization and chaotic adaptive real-coded biogeography-based optimization. Simulation, 2016. 92(9): p. 873-885

[11] Phillips, P., Intelligent facial emotion recognition based on stationary wavelet entropy and Jaya algorithm. Neurocomputing, 2018. 272: p. 668676

[12] Li, P. and G. Liu, Pathological Brain Detection via Wavelet Packet Tsallis Entropy and Real-Coded Biogeography-based Optimization. Fundamenta Informaticae, 2017. 151(1-4): p. 275-291

[13] Li, Y. and C. Cattani, Detection of Dendritic Spines using Wavelet Packet Entropy and Fuzzy Support Vector Machine. CNS \& Neurological Disorders - Drug Targets, 2017. 16(2): p. 116-121

[14] Nayak, D.R., Detection of unilateral hearing loss by Stationary Wavelet Entropy. CNS \& Neurological Disorders - Drug Targets, 2017. 16(2): p. $15-24$

[15] Evans, F., Haar wavelet transform based facial emotion recognition. Advances in Computer Science Research, 2017. 61: p. 342-346

[16] Lu, H.M., Facial Emotion Recognition Based on Biorthogonal Wavelet Entropy, Fuzzy Support Vector Machine, and Stratified Cross Validation. IEEE Access, 2016. 4: p. 8375-8385

[17] Zhan, T.M. and Y. Chen, Multiple Sclerosis Detection Based on Biorthogonal Wavelet Transform, RBF Kernel Principal Component 
Analysis, and Logistic Regression. IEEE Access, 2016. 4: p. 7567-7576

[18] Bouadjenek, N., H. Nemmour, and Y. Chibani, Fuzzy integrals for combining multiple SVM and histogram features for writer's gender prediction. IET Biometrics, 2017. 6(6): p. 429-437

[19] Chau, G. and G. Kemper, One Channel Subvocal Speech Phrases Recognition Using Cumulative Residual Entropy and Support Vector Machines. IEEE Latin America Transactions, 2015. 13(7): p. 2135-2143

[20] Rao, R.V., Abnormal Breast Detection in Mammogram Images by Feedforward Neural Network trained by Jaya Algorithm. Fundamenta Informaticae, 2017. 151: p. 191-211

[21] Lu, Z., A Pathological Brain Detection System Based on Radial Basis Function Neural Network. Journal of Medical Imaging and Health Informatics, 2016. 6(5): p. 1218-1222

[22] Yang, J., Identification of green, Oolong and black teas in China via wavelet packet entropy and fuzzy support vector machine. Entropy, 2015. 17(10): p. 6663-6682

[23] Liu, A., Magnetic resonance brain image classification via stationary wavelet transform and generalized eigenvalue proximal support vector machine. Journal of Medical Imaging and Health Informatics, 2015. 5(7): p. $1395-1403$

[24] Yang, J., Preclinical diagnosis of magnetic resonance (MR) brain images via discrete wavelet packet transform with Tsallis entropy and generalized eigenvalue proximal support vector machine (GEPSVM). Entropy, 2015. 17(4): p. 1795-1813

[25] Gorriz, J.M. and J. Ramírez, Wavelet entropy and directed acyclic graph support vector machine for detection of patients with unilateral hearing loss in MRI scanning. Frontiers in Computational Neuroscience, 2016. 10: Article ID. 160

[26] Zhou, X.-X., Comparison of machine learning methods for stationary wavelet entropy-based multiple sclerosis detection: decision tree, $k$ nearest neighbors, and support vector machine. Simulation, 2016. 92(9): p. 861-871

[27] Chen, M., Morphological analysis of dendrites and spines by hybridization of ridge detection with twin support vector machine. PeerJ, 2016. 4: Article ID. e2207

[28] Yang, M., Dual-Tree Complex Wavelet Transform and Twin Support Vector Machine for Pathological Brain Detection. Applied Sciences, 2016. 6(6): Article ID. 169

[29] Chen, S., J.-F. Yang, and P. Phillips, Magnetic resonance brain image classification based on weighted-type fractional Fourier transform and nonparallel support vector machine. International Journal of Imaging Systems and Technology, 2015. 25(4): p. 317-327

[30] Liu, G., Pathological brain detection in MRI scanning by wavelet packet Tsallis entropy and fuzzy support vector machine. SpringerPlus, 2015. 4(1): Article ID. 716

[31] Phillips, P., A Comprehensive Survey on Fractional Fourier Transform. Fundamenta Informaticae, 2017. 151(1-4): p. 1-48

[32] Senanayaka, J.S.L., H.V. Khang, and K.G. Robbersmyr. Towards online bearing fault detection using envelope analysis of vibration signal and decision tree classification algorithm. in 20th International Conference on Electrical Machines and Systems (ICEMS). 2017. Sydney, NSW, Australia: IEEE. p. 1-6

[33] Chakraborty, S., S.K. Adhikary, and M. Roy. Automatic land-cover classification using semi-supervised multilayer perceptron for analyzing remotely sensed images. in International Conference on Innovations in Electronics, Signal Processing and Communication (IESC). 2017. Shillong, India: IEEE. p. 33-38

[34] Zhao, G. Polarimetric synthetic aperture radar image segmentation by convolutional neural network using graphical processing units. Journal of Real-Time Image Processing, 2017, DOI: 10.1007/s11554-017-07170 .

[35] Lv, Y.-D., Alcoholism detection by data augmentation and convolutional neural network with stochastic pooling. Journal of Medical Systems, 2018. 42(1): Article ID. 2

[36] Muhammad, K. Image based fruit category classification by 13-layer deep convolutional neural network and data augmentation. Multimedia Tools and Applications, 2017, DOI: 10.1007/s11042-017-5243-3

[37] Bibin, D., M.S. Nair, and P. Punitha, Malaria Parasite Detection From
Peripheral Blood Smear Images Using Deep Belief Networks. IEEE Access, 2017. 5: p. 9099-9108

[38] Jia, W., Three-Category Classification of Magnetic Resonance Hearing Loss Images Based on Deep Autoencoder. Journal of Medical Systems, 2017. 41(10): Article ID. 165

[39] Hou, X.-X. Seven-layer deep neural network based on sparse autoencoder for voxelwise detection of cerebral microbleed. Multimedia Tools and Applications, 2017, DOI: 10.1007/s11042-017-4554-8. 